\title{
Movimento de partículas-teste no espaço-tempo de Reissner-Nordström
}

\author{
Test-particles motion in Reissner-Nordström spacetime
}

\author{
João P. B. Brito ${ }^{1}$, Rafael P. Bernar ${ }^{1,2}$, Carolina L. Benone ${ }^{3}$, Luís C. B. Crispino*1] \\ ${ }^{1}$ Universidade Federal do Pará, Faculdade de Física, Belém, PA, Brasil. \\ ${ }^{2}$ Universidade Federal do Maranhão, Departamento de Física, São Luís, MA, Brasil. \\ ${ }^{3}$ Universidade Federal do Pará, Faculdade de Física, Salinópolis, PA, Brasil.
}

\begin{abstract}
Recebido em 08 de janeiro de 2020. Revisado em 18 de fevereiro de 2020. Aceito em 24 de fevereiro de 2020.
O movimento de partículas-teste pode ser analisado usando-se as ferramentas da Teoria da Relatividade Geral. Algumas verificações clássicas desta teoria envolvem trajetórias de partículas-teste no espaço-tempo esfericamente simétrico associado à solução de Schwarzschild. Um outro espaço-tempo com esta simetria é o descrito pela solução de Reissner-Nordström, associada a uma distribuição esférica de massa e carga elétrica como, por exemplo, um buraco negro carregado. Neste espaço-tempo, analisamos o movimento de partículas livres, as quais seguem geodésicas, e de partículas com carga elétrica que, portanto, interagem eletricamente com o objeto central.

Palavras-chave: Espaços-Tempos Esfericamente Simétricos, Solução de Reissner-Nordström, Geodésicas, Trajetórias de Partículas.
\end{abstract}

\begin{abstract}
Test-particles motion can be analyzed using the tools of the Theory of General Relativity. Some classical verifications of this theory involve test-particles trajectories in the spherically symmetric spacetime associated to the Schwarzschild solution. Another spacetime with this symmetry is the one described by the Reissner-Nordström solution, associated to a spherical distribution of mass and electric charge as, for instance, a charged black hole. In this spacetime, we analyze the motion of free particles, which follow geodesics, and of particles with electric charge, which, therefore, interact electrically with the central object.

Keywords: Spherically Symmetric Spacetimes, Reissner-Nordström Solution, Geodesics, Particles Trajectories.
\end{abstract}

\section{Introdução}

A Teoria da Relatividade Geral (TRG) descreve a gravitação de maneira intrinsecamente relacionada à geometria do espaço-tempo. Por sua vez, a geometria do espaço-tempo é descrita por um campo tensorial, denominado tensor métrico, ou, simplesmente, métrica. $\mathrm{O}$ tensor métrico é determinado pelo conteúdo de matéria e energia presente no espaço-tempo, por meio das equações de campo da TRG (também chamadas de equações de campo de Einstein ou, simplesmente, equações de Einstein).

Hans Jacob Reissner e Gunnar Nordström, em 1916 1] e 1918 [2], respectivamente, obtiveram de forma independente uma solução exata das equações de campo de Einstein. A solução de Reissner-Nordström (RN), como ficou conhecida, descreve o espaço-tempo ao redor de uma distribuição esfericamente simétrica com massa e carga elétrica possuindo, portanto, um campo elétrico diferente de zero. No cenário em que a carga elétrica é zero, a solução de RN se reduz à solução encontrada pelo físico e astrônomo alemão Karl Schwarzschild e publicada em 1916 [3]. A solução de Schwarzschild, associada a uma

*Endereço de correspondência: crispino@ufpa.br fonte puntual de matéria, foi a primeira solução exata das equações de Einstein. (Para uma revisão histórica da solução de Schwarzschild, ver, e.g., Ref. 4].)

As soluções de RN e Schwarzschild, além de descreverem o espaço-tempo exterior a uma distribuição esférica de massa, com ou sem carga elétrica, respectivamente, também podem descrever o espaço-tempo de buracos negros estáticos com simetria esférica. Um buraco negro possui uma fronteira de não retorno, a qual é uma hipersuperfície nula ${ }^{1}$, denominada horizonte de eventos.

O buraco negro de RN é uma solução mais geral que a de Schwarzschild e pode apresentar um ou dois horizontes: um horizonte de eventos (mais externo) e outro, denominado de Cauchy ${ }^{2}$ (mais interno), assemelhando-se à estrutura dos horizontes nos buracos negros de Kerr,

\footnotetext{
${ }^{1}$ Uma hipersuperfície é nula se um vetor ortogonal a ela é nulo (neste caso, é também um vetor tangente a ela) 5, 6.

${ }^{2} \mathrm{O}$ horizonte de Cauchy é uma hipersuperfície nula e o futuro de um observador que a atravessa não é determinado pela sua história fora deste horizonte 5 7, 7 . Entretanto, pequenas perturbações de campos-teste na geometria de RN (e.g. perturbações do campo eletromagnético) se tornam singulares no horizonte de Cauchy 9]. Acredita-se que tais instabilidades nos campos-teste sejam reflexo de que o horizonte de Cauchy se torna uma singularidade intrínseca, quando a teoria não-linear completa é considerada 10 .
} 
tidos na literatura como os buracos negros mais comuns na natureza. O objeto compacto no centro da Via Láctea é um candidato a um buraco negro girante supermassivo 11]. Recentemente, a coleta de dados de uma rede de radiotelescópios, espalhados ao redor do globo, possibilitou a observação da sombra de um buraco negro supermassivo, com mais de seis bilhões de massas solares, a aproximadamente 53 milhões de anos-luz de distância de nós, no centro da galáxia M87 12 17]. (Mais detalhes sobre sombras de buracos negros podem ser encontrados na Ref. [18.)

Muitas características do espaço-tempo podem ser analisadas estudando o movimento de partículas-teste. Algumas verificações clássicas da TRG envolvem trajetórias de partículas-teste: $(i)$ a precessão do periélio do planeta Mercúrio, cujos dados observacionais foram usados para checar a consistência da TRG $[19,20] ;(i i)$ a deflexão da luz que passa próximo ao Sol, fenômeno que foi originalmente medido a partir de observações no Brasil e na costa oeste africana, em 1919, durante um eclipse Solar 21 23] ( iii) o redshift (desvio para o vermelho) gravitacional, que foi medido com sucesso primeiramente por R. V. Pound e G. A. Rebka em 1960 25 4 Nestas verificações, pode-se considerar, com boa aproximação, que os objetos-fonte encurvam o espaço-tempo de acordo com a solução de Schwarzschild.

Trajetórias de partículas em espaços-tempos de buracos negros foram estudadas de forma abrangente na literatura. Em particular, no caso do espaço-tempo de RN, destacamos as Refs. 27-29] e, para uma abordagem detalhada das órbitas circulares de partículas carregadas neste espaço-tempo, destacamos as Refs. 30,31. Neste artigo, como uma proposta de abordagem acessível a um público com conhecimento básico da TRG, exploramos a geometria de RN estudando as trajetórias geodésicas 5 e as trajetórias de partículas carregadas neste espaçotempo.

O restante deste trabalho é composto da seguinte maneira: Na Seção 2, descrevemos o espaço-tempo esfericamente simétrico de um buraco negro carregado e estático. Na Seção 3 analisamos as equações de movimento para partículas livres neutras (geodésicas) e para partículas carregadas e discutimos alguns aspectos das trajetórias, tais como sua estabilidade. Na Seção 4 tratamos das

\footnotetext{
${ }^{3}$ Este teste também foi proposto na Ref. 24], na qual o encurvamento da trajetória de uma onda eletromagnética é verificado enviando ondas de rádio da Terra para Vênus ou Mercúrio, quando ambos passam por detrás do Sol.

${ }^{4} \mathrm{O}$ redshift gravitacional foi verificado recentemente por Abuter et al. 26 na órbita de uma estrela no entorno do objeto compacto no centro da galáxia Via Láctea (Sagittarius $A^{*}$ ). Conforme a estrela aproxima-se de seu periastro ( $\approx 120$ unidades astronômicas), ponto da trajetória onde o campo gravitacional do astro se torna mais intenso, um aumento no comprimento de onda de seu espectro de emissão é observado.

${ }^{5}$ Os caminhos seguidos por partículas livres são geodésicas do espaço-tempo. As geodésicas tipo-tempo são as trajetórias de partículas massivas (e sem carga elétrica, no caso do espaço-tempo de $\mathrm{RN}$ ) e as geodésicas nulas (tipo-luz) são as trajetórias de partículas não-massivas.
}

órbitas circulares. Na Seção 5 , analisamos as trajetórias de partículas-teste no espaço-tempo de RN, assim como alguns aspectos da seção de choque de absorção de um buraco negro carregado, no limite geométrico. Na Seção 6, apresentamos nossas discussões finais. Adotamos unidades geometrizadas nas quais a velocidade da luz $c$ e a constante gravitacional newtoniana $G$ são definidas iguais à unidade e consideramos a assinatura $(+,-,-,-)$ para a métrica do espaço-tempo.

\section{O Espaço-Tempo de Reissner-Nordström}

Nesta Seção, apresentamos um resumo sobre espaçostempos esfericamente simétricos e permeados por um campo elétrico estático, abordando algumas das suas propriedades principais.

As equações de Einstein, em um espaço-tempo que possui um campo elétrico, podem ser escritas com ${ }^{6}$

$$
R_{\mu \nu}=2\left(F_{\mu \lambda} F_{\nu}{ }^{\lambda}-\frac{1}{4} g_{\mu \nu} F_{\lambda \sigma} F^{\lambda \sigma}\right)
$$

onde $F_{\mu \nu}$ são as componentes covariantes do tensor eletromagnético [36], $R_{\mu \nu}$ são as componentes do tensor de Ricci e $g_{\mu \nu}$ são as componentes da métrica.7

As componentes $F_{\mu \nu}$ se relacionam com o quadrivetor potencial $A_{\mu}$ por 36 .

$$
F_{\mu \nu}=\partial_{\mu} A_{\nu}-\partial_{\nu} A_{\mu}
$$

As equações de Maxwell não triviai:8 na região sem fonte, são dadas por

$$
\nabla_{\mu} F^{\mu \nu}=0
$$

Supondo que tanto a métrica quanto o campo eletromagnético possuem simetria esférica, é possível resolver o sistema dado pelas Eqs. (1) e (3), obtendo-se as componentes $A_{\mu}$, a saber $8,33,35$ :

$$
A_{t}=\frac{Q}{r}, A_{r}=A_{\theta}=A_{\phi}=0,
$$

em que $Q$ é a carga (em unidades gaussianas) da fonte elétrica e gravitacional (uma estrela carregada ou um buraco negro carregado, por exemplo).

O elemento de linha da solução de $\mathrm{RN}$ pode ser escrito como

$$
d s^{2}=f(r) d t^{2}-\frac{1}{f(r)} d r^{2}-r^{2}\left(d \theta^{2}+\sin ^{2} \theta d \phi^{2}\right),
$$

\footnotetext{
${ }^{6}$ Assumimos que o leitor possui conhecimentos básicos da álgebra tensorial e variedades riemannianas. Como bibliografia para esses temas, podemos citar as Refs. $5,6,8,32,35$.

${ }^{7}$ Os índices de letras gregas variam de 0 a 3 , e denotam as quatro coordenadas. $O$ índice 0 representa a coordenada temporal e os índices de 1 a 3, as coordenadas espaciais.

${ }^{8}$ As equações de Maxwell dadas por $\nabla_{[\alpha} F_{\mu \nu]}=0$ são automaticamente satisfeitas por $F_{\mu \nu}$, dado pela Eq. 2 .
} 
em que

$$
f(r)=\left(1-\frac{r_{+}}{r}\right)\left(1-\frac{r_{-}}{r}\right)
$$

com

$$
r_{ \pm} \equiv M \pm \sqrt{M^{2}-Q^{2}}
$$

A quantidade $M$ é a massa da fonte gravitacional. Nos cenários em que a solução (5) é válida para todo $r>0$ e $M^{2} \geq Q^{2}$, temos um espaço-tempo de um buraco negro carregadd 9 Neste caso, $r_{+}$é a posição radial do horizonte de eventos e $r_{-}$a posição radial do horizonte de Cauchy. No caso em que o buraco negro é descarregado $(|Q| \rightarrow 0)$, temos os limites $r_{-} \rightarrow 0$ e $r_{+} \rightarrow 2 M$. No caso em que o buraco negro é extremamente carregado $(|Q| \rightarrow M)$, temos os limites $r_{ \pm}^{e} \rightarrow M$.

O sistema de coordenadas usado na Eq. (5) é problemático nas posições em que $f(r)=0$ (sobre os horizontes). Já em $r=0$, há uma singularidade intrínseca, como mostra o escalar de Kretschmann [37, qual seja

$$
K_{Q} \equiv R_{\mu \nu \rho \sigma} R^{\mu \nu \rho \sigma}=\frac{8\left(7 Q^{4}-12 M Q^{2} r+6 M^{2} r^{2}\right)}{r^{8}}
$$

onde $R_{\mu \nu \rho \sigma}$ são as componentes covariantes do tensor de Riemann e $K_{Q}$ é o escalar de Kretschmann para o espaço-tempo de RN.

Na região $r<7 Q^{2} / 12 M$, o escalar de Kretschmann é maior para $|Q| \neq 0$, se comparado com o caso em que $|Q|=0$. Isto é devido à contribuição negativa da carga $Q$ para o escalar $K_{Q}$, como ilustrado na Fig. 1

Analisando as geodésicas radiais nulas, para as quais $d s=d \theta=d \phi=0$, no elemento de linha dado pela Eq. (5), podemos construir um diagrama dos cones de

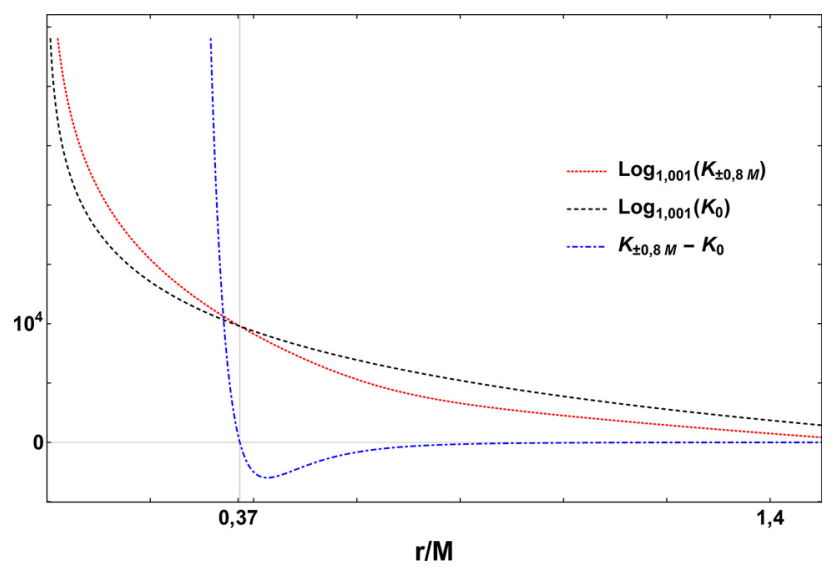

Figura 1: Escalar de Kretschmann $K_{Q}$, dado pela Eq. (8), para espaços-tempos com $|Q|=0$ (Schwarzschild) e com $|Q|=0,8 M$ (RN), e a contribuição da carga elétrica, $K_{ \pm 0,8 M}-K_{0}$, que é negativa para $r \gtrsim 0,37 M$.

\footnotetext{
${ }^{9}$ Se o objeto central for uma estrela carregada eletricamente, por exemplo, então o espaço-tempo no interior da estrela não é descrito pela solução de RN. Neste caso, a solução dada pela Eq. 5 é válida somente para $r \geq r_{\text {estrela }}$, em que $r_{\text {estrela }}$ é a posição radial da superfície da estrela.
}

lut 10 do espaço-tempo de RN, nas coordenadas $t$ e $r$. Omitindo as coordenadas angulares $\theta$ e $\phi$, os cones de luz são construídos a partir da interseção das geodésicas tipo-luz ingoing (que tendem a entrar no buraco negro) e outgoing (que tendem a sair do buraco negro), como ilustrado na Fig. 2, de forma que dentro do cone de luz residem os caminhos tipo-tempo.

Da Fig. 2, podemos observar que, em decorrência do fato de o espaço-tempo ser assintoticamente plano, para valores de $r \gg r_{+}$, as geodésicas tipo-luz se aproximam de retas com inclinação de $\pm 45^{\circ}$, como ocorre globalmente no espaço-tempo plano minkowskiano, para o sistema de coordenadas correspondente. Para um observador estático no infinito espacial: (i) as geodésicas demoram um tempo infinito para cruzar o horizonte de eventos, (ii) um raio de luz que emerge do horizonte de eventos sofre um "redshift infinito", e (iii) um objeto massivo que cai no buraco negro parece diminuir sua velocidade a zero à medida que se aproxima do horizonte de eventos. (Estas conclusões estão associadas ao sistema de coordenadas naturais deste observador estático no infinito.) No entanto, uma partícula massiva que cai em direção ao buraco negro atravessa o horizonte em um valor finito de seu tempo próprio. Dentro da hipersuperfície $r=r_{+}$, todas as trajetórias tipo-tempo e nulas são obrigadas a seguir na direção de $r=r_{-}$. A singularidade é uma linha tipo-tempo e as geodésicas, exceto as geodésicas radiais nulas, terminam antes de alcançar a singularidade 8.

\section{Equações de Movimento de Partículas-teste}

Nesta Seção, analisamos as trajetórias geodésicas tipotempo (tipo-luz ou nula) no espaço-tempo de RN, que são as órbitas de partículas massivas (não-massivas) livres

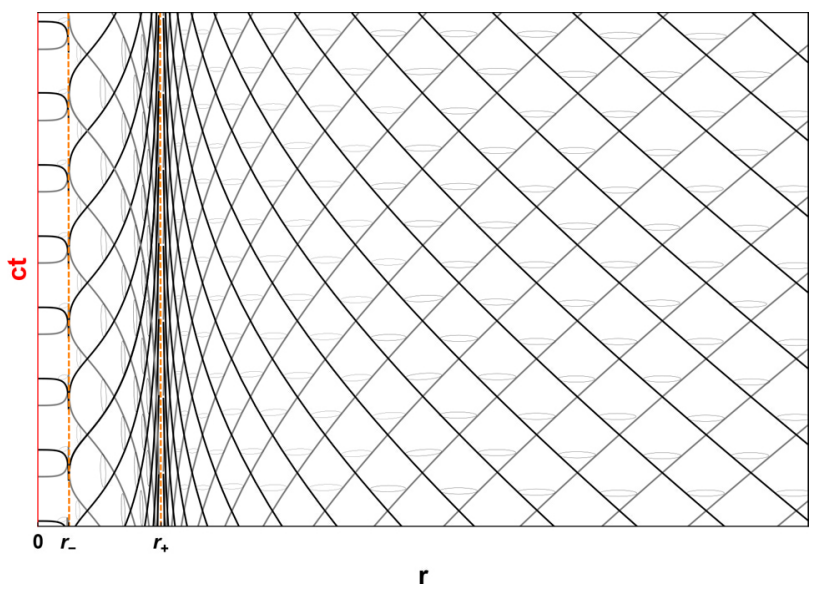

Figura 2: Diagrama ilustrando os cones de luz no espaço-tempo de RN, descrito pela Eq. (5), com as coordenadas angulares suprimidas. Nesta figura, escolhemos $|Q|=0,8 M$.

${ }^{10} \mathrm{O}$ cone de luz, para um dado evento, é o conjunto de caminhos possíveis no espaço-tempo que podem ser seguidos por partículas não-massivas e que passam pelo evento em questão. 
e, portanto, neutras. Analisamos também as trajetórias de partículas eletricamente carregadas. Consideramos partículas-teste ${ }^{11}$ massivas, com massa de repouso $m$ e carga elétrica $q$. Nossa análise inclui o caso $|Q|=0$ (espaço-tempo de Schwarzschild).

As partículas carregadas interagem gravitacional e eletricamente com o buraco negro carregado. A lagrangiana para uma partícula carregada, na presença do quadrivetor potencial $A_{\mu}$, pode ser escrita como $8,30,12$

$$
L=\frac{1}{2} g_{\mu \nu} \frac{d x^{\mu}}{d \lambda} \frac{d x^{\nu}}{d \lambda}+\frac{q}{m} A_{\mu} \frac{d x^{\mu}}{d \lambda}
$$

onde $\lambda$ é um parâmetro afim. 13

Por meio das equações de Euler-Lagrange [40], a saber

$$
\frac{\partial L}{\partial x^{\sigma}}-\frac{d}{d \lambda}\left(\frac{\partial L}{\partial \dot{x}^{\sigma}}\right)=0,
$$

obtemos a equação de movimento de uma partícula carregada, que sofre a ação da força de Lorentz, no espaçotempo de RN, qual seja

$$
\frac{d^{2} x^{\mu}}{d \lambda^{2}}+\Gamma_{\rho \sigma}^{\mu} \frac{d x^{\rho}}{d \lambda} \frac{d x^{\sigma}}{d \lambda}=\frac{q}{m} F_{\nu}^{\mu} \frac{d x^{\nu}}{d \lambda} .
$$

Os símbolos de Christoffel $\Gamma^{\mu}{ }_{\rho \sigma}$ são dados por $[5,6,8,32$ 35 .

$$
\Gamma_{\rho \sigma}^{\mu}=\frac{1}{2} g^{\mu \lambda}\left(\partial_{\rho} g_{\lambda \sigma}+\partial_{\sigma} g_{\rho \lambda}-\partial_{\lambda} g_{\rho \sigma}\right) .
$$

Na Eq. (11), com $A_{\mu}$ dado pela Eq. (4), se $q Q=0$, recobramos a equação da geodésica; e se $q Q \neq 0$, as partículas não seguem geodésicas do espaço-tempo. A interação entre as cargas elétricas do buraco negro e da partícula-teste, expressa pelo lado direito da Eq. (11), altera as trajetórias das partículas-teste.

Devido à simetria do espaço-tempo de RN, existem as seguintes quantidades conservadas:

$$
p_{t}=f(r) \dot{t}+\frac{q Q}{m r} \equiv \frac{E}{m} \text { e } p_{\phi}=-r^{2} \sin ^{2} \theta \dot{\phi} \equiv-\frac{l}{m},
$$

${ }^{11}$ Uma partícula-teste é aquela que não influencia a geometria do espaço-tempo no qual se move. Além disso, assumimos que o sistema não perde energia pela emissão de ondas gravitacionais ou eletromagnéticas 38.

${ }^{12} \mathrm{~A}$ lagrangiana relativística para uma partícula carregada, na verdade, tem a forma

$$
L=-m \sqrt{g_{\mu \nu} \frac{d x^{\mu}}{d \lambda} \frac{d x^{\nu}}{d \lambda}}+q A_{\mu} \frac{d x^{\mu}}{d \lambda} .
$$

A ação, $S=\int L d \lambda$, proveniente da lagrangiana acima, é invariante por reparametrização, isto é, sob a transformação $\lambda \rightarrow \lambda^{\prime}(\lambda) 39$. Isto nos permite escrever uma lagrangiana modificada, dada pela Eq. (9), a qual é mais conveniente para calcularmos as equações de movimento, as mesmas associadas à lagrangiana original. A lagrangiana 9 pode ser encontrada nas referências clássicas sobre o tema (cf. Eq. (103) do capítulo 5 (p. 224) da Ref. 8] e Eq. (3) da Ref. [30]).

${ }^{13}$ Dada uma curva geodésica $x^{\mu}(\lambda)$, o parâmetro $\lambda$ é dito afim se o vetor tangente à curva, em relação ao parâmetro $\lambda$, é o mesmo em todos os pontos ao longo da curva, i.e. o vetor tangente é paralelamente transportado ao longo da curva 32 35. onde o ponto sobre as letras denota diferenciação em relação ao parâmetro afim $\lambda$. As quantidades $E / m$ e $l / m$ são interpretadas como a energia e a magnitude do momento angular da partícula por unidade de massa, respectivamente, medidas por um observador estático no infinito. Temos também a constante devido à normalização da quadrivelocidade ao longo do movimento, dada por

$$
\epsilon \equiv g_{\mu \nu} \frac{d x^{\mu}}{d \lambda} \frac{d x^{\nu}}{d \lambda}
$$

No caso de partículas massivas, podemos adotar o tempo próprio $\tau$ (tempo medido no referencial instantaneamente em repouso da partícula) como parâmetro afim $\lambda$ e, portanto, teremos $\epsilon=1$ nas unidades adotadas 14 Para partículas não-massivas, temos que $\epsilon=0$. Notemos que a Eq. (13), com $q=0$, continua sendo válida para partículas não-massivas, desde que as substituições $E / m \rightarrow E$ e $l / m \rightarrow l$ sejam feitas. Portanto, a menos que assumido o contrário, as equações das Seções 3 e 4 válidas para trajetórias de partículas massivas, são também válidas para partículas não-massivas, sendo suficiente fazermos $q=0$ e $E / m \rightarrow E, l / m \rightarrow l$.

Das Eqs. 11 e 13, temos que a equação de movimento para a coordenada $\theta$ é dada por

$$
\ddot{\theta}=\frac{l^{2} \cos \theta}{m^{2} r^{4} \sin ^{3} \theta}-\frac{2 \dot{r} \dot{\theta}}{r} .
$$

Podemos assumir, sem perda de generalidade, que o movimento acontece no plano equatorial, isto é, $\theta=\pi / 2$, de forma que $\dot{\theta}=0$ e $\ddot{\theta}=0$, em acordo com a equação de movimento 15. De fato, como o espaço-tempo de RN é esfericamente simétrico, o plano do movimento sempre pode ser transformado no plano equatorial por uma rotação dos eixos das coordenadas espaciais. Notemos, portanto, que o movimento da partícula sempre acontece em um plano que atravessa o centro do objeto fonte da interação gravitacional.

As trajetórias de partículas podem ser descritas por uma única equação de balanço de energia. A lagrangiana $(9)$ e as constantes dadas nas Eqs. (13) e (14) nos permitem escrever esta equação de balanço de energia como

$$
\dot{r}^{2}+f(r)\left(\epsilon+\frac{l^{2}}{m^{2} r^{2}}\right)=\left(\frac{E}{m}-\frac{q Q}{m r}\right)^{2} .
$$

Da Eq. 16], obtemos a energia da partícula nas posições em que $\dot{r}=0$, qual seja

$$
\frac{E^{ \pm}}{m}=\frac{q Q}{m r} \pm \sqrt{f(r)\left(\epsilon+\frac{l^{2}}{m^{2} r^{2}}\right)} .
$$

Neste trabalho, nos restringimos aos cenários em que a energia da partícula é $E \equiv E^{+}[31]$. Nota-se que, no limite $r \rightarrow \infty$, temos $E^{ \pm} \rightarrow \pm m$ e fazendo $q \rightarrow-q$, temos $E^{+} \rightarrow-E^{-}$.

\footnotetext{
${ }^{14}$ Caso não tivéssemos adotado unidades nas quais $c=1$, teríamos $\epsilon=c^{2}$.
} 
Definimos o potencial efetivo das trajetórias geodésicas como

$$
V_{e f f}^{T R G} \equiv \frac{1}{2} f(r)\left(\epsilon+\frac{l^{2}}{m^{2} r^{2}}\right)
$$

No limite $r \rightarrow \infty$, temos $V_{e f f}^{T R G} \rightarrow 1 / 2(\rightarrow 0)$ para partículas massivas (não-massivas).

Na Fig. 3. comparamos os potenciais efetivos de partículas neutras na TRG e na teoria newtoniana para a gravitação, cujo potencial efetivo pode ser escrito como 41

$$
V_{e f f}^{\mathrm{Newton}} \equiv-\epsilon \frac{M}{r}+\frac{l^{2}}{2 m^{2} r^{2}} .
$$

Nos extremos do potencial efetivo ocorrem órbitas circulares e nos pontos de inflexão ocorrem as órbitas
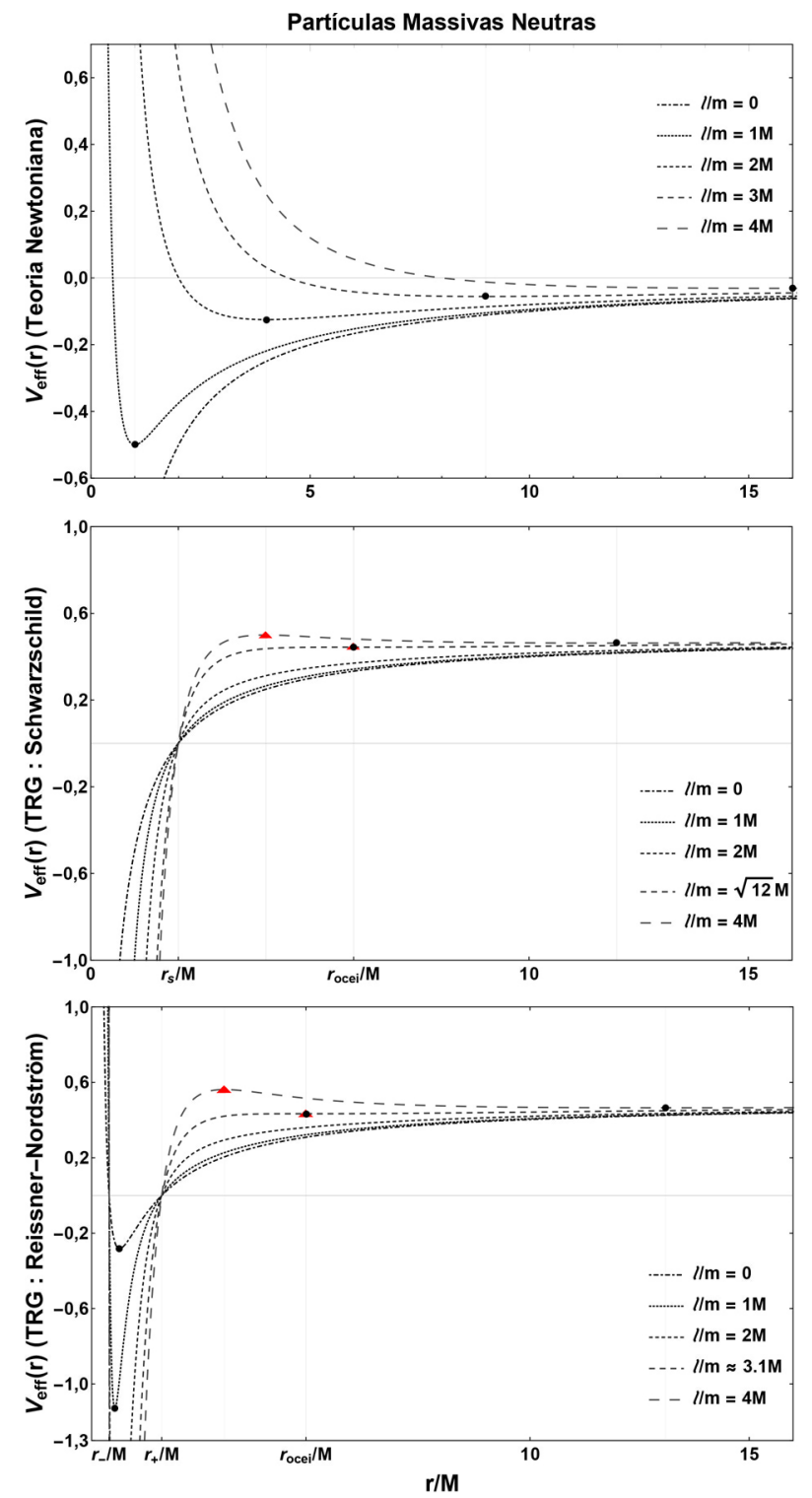

circulares estáveis mais internas, definidas pela posição radial $r_{o c e i}$. As órbitas estáveis (pontos de mínimo do potencial) ocorrem na região $r \geq r_{o c e i}$. Na região $r<r_{o c e i}$, ocorrem apenas órbitas instáveis (pontos de máximo do potencial). Na Fig. 4 mostramos o comportamento de $r_{\text {ocei }}$ em função de $|\vec{Q}| / M$.

Os pontos de máximo e mínimo do potencial, representados na Fig. 3 como triângulos e discos, respectivamente, aproximam-se de $r_{\text {ocei }}$ à medida que o momento angular decresce até um valor mínimo (em que os pontos coincidem na posição $r_{o c e i}$ ), além do qual não são permitidas órbitas circulares em qualquer região (não há extremos de $\left.V_{e f f}^{T R G}\right)$. Neste caso, uma partícula se movendo em direção ao buraco negro $(\dot{r}<0)$ não encontra uma barreira de potencial e, inevitavelmente, cruzará o horizonte de
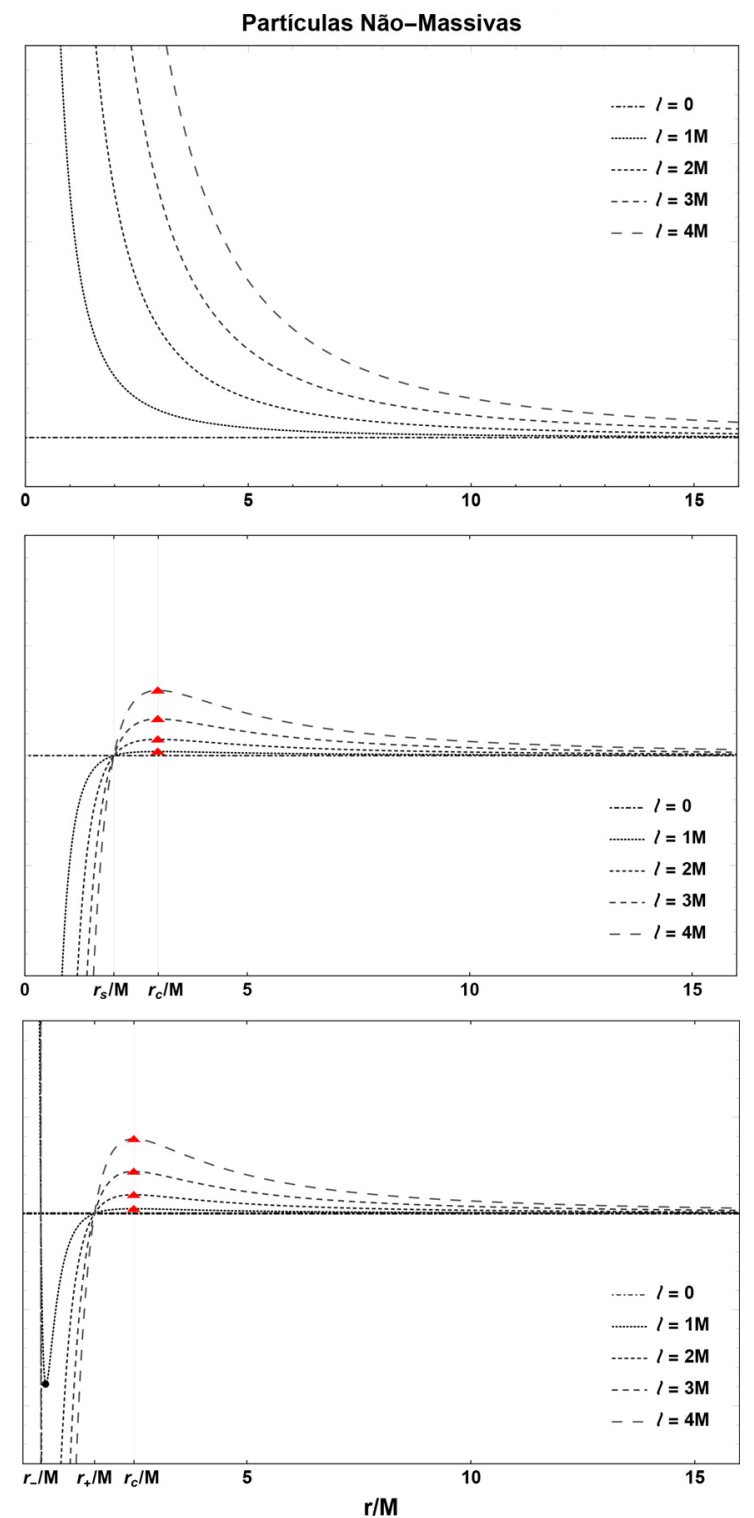

Figura 3: Potenciais efetivos de partículas massivas neutras (esquerda) e não-massivas (direita) na gravitação newtoniana e na TRG, para diferentes valores do momento angular por unidade de massa da partícula-teste. Os triângulos vermelhos e os discos pretos denotam os pontos de máximo e mínimo, respectivamente. O buraco negro de RN possui $|Q|=0,8 M$. Na posição $r_{\text {ocei }}$ está localizada a órbita circular estável mais interna, na posição $r_{c}$ está localizada a geodésica circular nula e $r_{s}$ é o raio de Schwarzschild. 
eventos. No limite $l / m \rightarrow \infty$, temos o limite $r_{\text {ocei }} \rightarrow \infty$, e a órbita instável tendendo, assintoticamente, à geodésica nula circular, que ocorre na posição radial $r_{c}$. Os pontos de mínimo (representamos como discos na Fig. 3) entre os horizontes não indicam órbitas de partículas, uma vez que, para $r=$ constante, a curva é tipo-espaço nessa região. No contexto da gravitação newtoniana não existem órbitas circulares instáveis e as órbitas circulares estáveis estão localizadas na posição radial $r=l^{2} /\left(m^{2} M\right)$.

Vemos também na Fig. 3 que nas soluções de Schwarzschild e RN os potenciais têm, qualitativamente, a mesma forma até o horizonte de eventos (denotado por $r_{s}$ no caso de Schwarzschild e $r_{+}$no caso de RN), sobre o qual se anulam. No entanto, na região interna ao horizonte de eventos, os potenciais diferem notoriamente. Na solução de Schwarzschild, o potencial decresce até $-\infty$ tão mais rapidamente quanto maior o momento angular da partícula e, inevitavelmente, a partícula cai na singularidade. Na solução de RN, o potencial decresce até um ponto de mínimo global. $\mathrm{O}$ valor do potencial neste ponto de mínimo global é tanto menor ${ }^{15}$ quanto maior for o momento angular da partícula. No cenário de um buraco negro extremo, o ponto de mínimo global coincide com a posição do horizonte do buraco negro $\left(r_{ \pm}^{e}=M\right)$, em que $V_{e f f}^{T R G}=0$. Nesta posição, a geodésica circular nula é estável e coincide com as geodésicas geradoras da hipersuperfície nula [29]. Devido ao potencial crescer rapidamente próximo de $r_{-}$, as trajetórias tipo-tempo (e tipo-luz, se $l \neq 0$ ) têm pontos de retorno na região interna ao horizonte de Cauchy 42$]{ }^{16}$ No espaço-tempo de $\mathrm{RN}$, somente as geodésicas nulas radiais $(l=0)$ alcançam $r=0$ 8.

As trajetórias em torno do buraco negro podem ser classificadas de acordo com o seu comportamento em relação ao horizonte de eventos. As trajetórias denominadas de primeiro tipo não cruzam o horizonte de eventos e as denominadas de segundo tipo são aquelas que cruzam este horizonte. Entre as trajetórias de primeiro tipo, há órbitas ligadas (para as quais $E<m$ ) e não ligadas (para as quais $E \geq m$ ). A órbita circular limite associada às partículas com $E=m$ é denominada órbita circular ligada mais interna possível e ocorre na posição radial $r_{\text {ocli }}$, cujo comportamento em função de $|Q|$ é mostrado na Fig. 4 As órbitas ligadas podem oscilar entre duas posições radiais $r_{\min }$ (periápside) e $r_{\max }$ (apoápside). Já as órbitas não ligadas, as quais vêm do infinito, podem se aproximar do buraco negro até um raio mínimo e, em seguida, retornar ao infinito; ou podem tender, assintoticamente, a uma órbita circular instável.

As órbitas estáveis ocorrem na região acima da curva de $r_{\text {ocei }}$ na Fig. 4 e na região abaixo desta curva ocorrem apenas órbitas instáveis. Na região entre as curvas assinaladas por $r_{o c l i}$ e $r_{c}$, estão localizadas as geodésicas

\footnotetext{
15 Note que o potencial no ponto de mínimo global é negativo.

${ }^{16}$ As trajetórias podem retornar por $r_{-}$e $r_{+}$para um espaço-tempo assintoticamente plano diferente. Para uma explicação mais detalhada sobre este assunto, sugerimos as Refs. 7 .
}

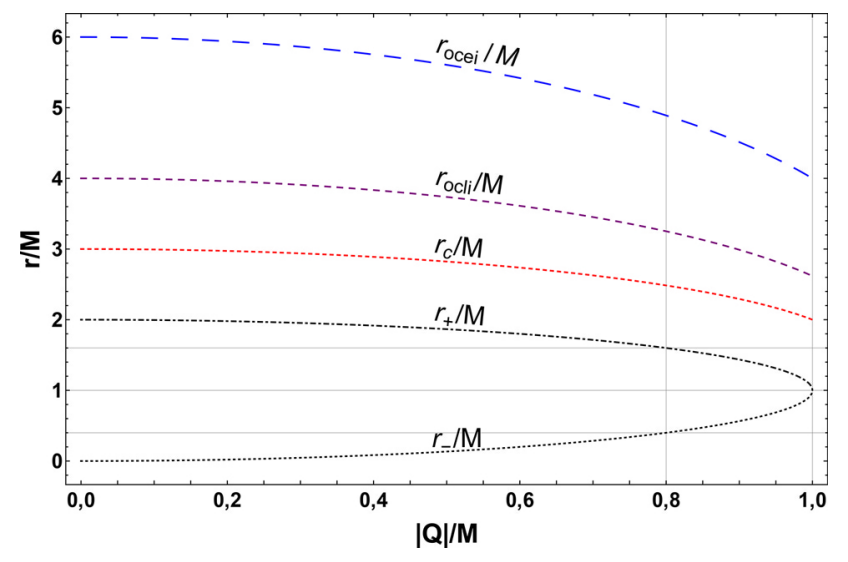

Figura 4: Posições radiais $r_{-}, r_{+}, r_{c}, r_{o c l i}$ e $r_{o c e i}$ em função da carga $|Q|$ do buraco negro. Na posição $r_{o c l i}$ ocorre a órbita circular ligada mais interna possível. As órbitas circulares $r_{o c l i}$ e $r_{\text {ocei }}\left(r_{c}\right)$ estão associadas às partículas massivas descarregadas (não-massivas).

circulares instáveis e não ligadas. Partículas nestas órbitas escaparão para o infinito (cairão no buraco negro) se perturbadas radialmente para fora (dentro). Na região entre $r_{c}$ e $r_{+}$, não há geodésicas circulares e sobre a curva assinalada por $r_{+}$, temos as geodésicas nulas geradoras do horizonte de eventos.

\section{4. Órbitas Circulares}

As órbitas circulares permitem um tratamento analítico detalhado devido à sua simplicidade. Além disso, tais órbitas possuem um papel importante na construção de outros tipos de trajetórias. Analisemos agora trajetórias circulares de partículas carregadas. Para órbitas circulares, temos que

$$
\dot{r}=\ddot{r}=0 .
$$

Derivando a Eq. (16) em relação a $r$, obtemos a seguinte expressão:

$$
\frac{l_{ \pm}^{2}}{m^{2}}=\frac{f(r)}{2 \bar{f}(r)^{2}}\left(\frac{q^{2}}{m^{2}} Q^{2} \pm \frac{|q Q|}{m} \sqrt{\zeta_{1}}\right)+\frac{M r-Q^{2}}{\bar{f}(r)},
$$

onde $\bar{f}(r) \equiv 2 Q^{2} / r^{2}-3 M / r+1, \zeta_{1} \equiv 4 \bar{f}(r) r^{2}+q^{2} Q^{2} / m^{2}$ e $l_{ \pm}$é identificado como o momento angular da partícula teste, sendo uma das quantidades conservadas ao longo das trajetórias circulares. Há duas soluções para o momento angular no caso de uma partícula carregada. Neste trabalho, consideramos $|q| / m \in] 0,1]$ e, nestes casos, as órbitas foram classificadas na Ref. 31. Nesta classificação, identifica-se a solução $l_{-}$da Eq. (21) como o momento angular das partículas com carga de sinal igual a do buraco negro e a solução $l_{+}$como o momento angular das partículas com carga de sinal oposto, para as órbitas circulares na região $r>r_{c}$, em que $r_{c}$, conforme mencionado anteriormente, é a posição radial da geodésica nula circular. 
Além do momento angular, destacamos também a energia da partícula em órbita circular, medida por um observador estático no infinito. Das Eqs. (17) e (21), obtemos

$$
\frac{E^{ \pm}}{m}=\frac{q Q}{m r}+\frac{f(r)}{\sqrt{2} r|\bar{f}(r)|} \sqrt{\zeta_{2} \pm \frac{|q Q|}{m} \sqrt{\zeta_{1}}},
$$

onde $\zeta_{2} \equiv 2 \bar{f}(r) r^{2}+q^{2} Q^{2} / m^{2}$.

No limite $q \rightarrow 0$, a Eq. (21) torna-se

$$
\frac{l^{2}}{m^{2}}=\frac{M r^{2}-Q^{2} r}{r-3 M+2 Q^{2} / r}
$$

e a Eq. 22 torna-se

$$
\frac{E}{m}=\frac{1-2 M / r+Q^{2} / r^{2}}{\left(1-3 M / r+2 Q^{2} / r^{2}\right)^{1 / 2}} .
$$

O momento angular e a energia de uma partícula massiva neutra em órbita circular, dados pelas Eqs. 23 e (24), respectivamente, aumentam quanto menor for a posição radial da órbita e diminuem quanto maior for a carga do buraco negro. No limite em que a órbita circular tende a $r_{c}$, ambas as quantidades, $E / m$ e $l / m$, divergem.

O comportamento do momento angular $l_{ \pm} / m$, dado pela Eq. 21), é apresentado nas Figs. 5 e 6 para um buraco negro com $Q=0,8 M$ e alguns valores de $q$. A força de Lorentz sobre as partículas com carga de mesmo sinal da carga do buraco negro é repulsiva e, portanto, compete com a interação gravitacional. Já a força de Lorentz sobre as partículas de carga de sinal oposto ao da carga do buraco negro é atrativa, somando-se à interação gravitacional e, neste caso, o momento angular necessário para a partícula manter-se na órbita circular aumenta à medida que o módulo da quantidade $\mathrm{q} / \mathrm{m}$ aumenta.

O momento angular, dado pela Eq. (23), é zero no caso em que $r \equiv r_{0}=Q^{2} / M$. A posição radial $r_{0}$ está localizada entre os horizontes. Porém, para valores da

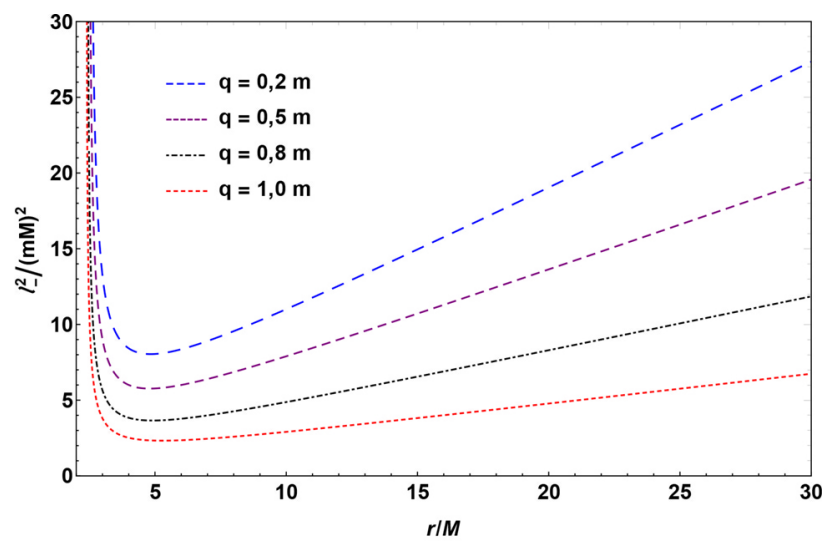

Figura 5: Momento angular, $l_{-}^{2} /\left(m^{2} M^{2}\right)$, de partículas com carga de sinal igual a do buraco negro, de acordo com a Eq. (21). O buraco negro possui carga $Q=0,8 M$.

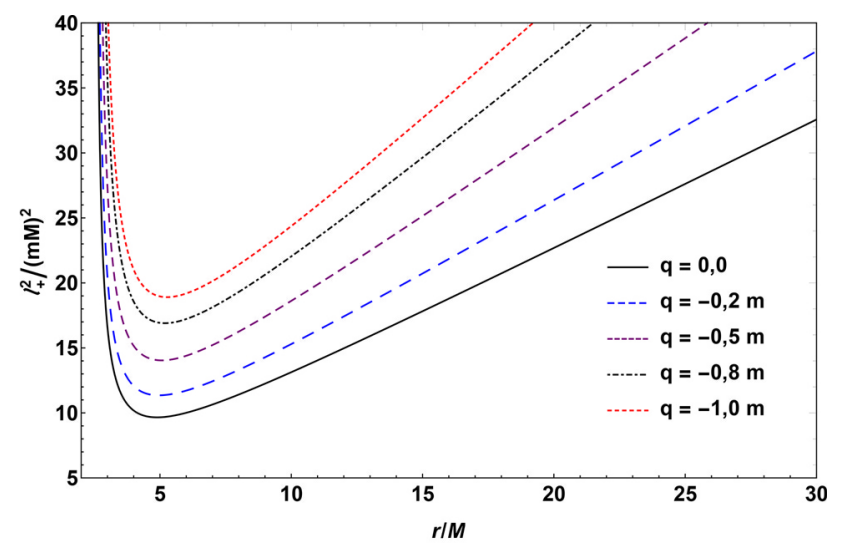

Figura 6: Momento angular, $l_{+}^{2} /\left(m^{2} M^{2}\right)$, de partículas com carga nula ou de sinal oposto a do buraco negro, de acordo com a Eq. (21). O buraco negro possui carga $Q=0,8 M$.

carga $|Q|>M$ (singularidade nua, i.e. não há horizontes enclausurando a singularidade), a partícula pode permanecer em $r_{0}$. Consequentemente, a matéria acretada pela singularidade, após perder energia suficiente, pode terminar nesta posição de equilíbrio [28].

No caso ideal, as partículas poderiam permanecer indefinidamente em órbitas circulares instáveis. Entretanto, em uma situação real, há muitas perturbações possíveis que podem fazer a partícula espiralar para fora (órbita de primeiro tipo) ou para dentro do buraco negro (órbita de segundo tipo) [5,8]. Estas perturbações podem ser, por exemplo, a pressão da radiação emitida pelo buraco negro ou a perda de energia através da emissão de ondas gravitacionais e/ou eletromagnéticas (no caso de partículas carregadas) pelo sistema [5].

A condição de estabilidade das órbitas circulares pode ser associada ao sinal da segunda derivada do potencial efetivo e o ponto de inflexão deste potencial fornece a posição radial da órbita circular estável mais interna $r_{\text {ocei }}$. Das Eqs. (16) e (17), define-se o seguinte potencial efetivo para as partículas carregadas [30]:

$$
V_{q} \equiv \frac{E}{m}=\frac{q Q}{m r}+\sqrt{f(r)\left(1+\frac{l^{2}}{m^{2} r^{2}}\right)} .
$$

Este potencial corresponde à energia por unidade de massa $E / m$ nos pontos em que $\dot{r}=0$ (e.g. ao longo das órbitas circulares). O potencial dado pela Eq. 25 está ilustrado na Fig. 7. O máximo do potencial $V_{q}$ é tanto maior quanto maior for $q Q / m$ (e menor é a seção de choque de absorção clássica das partículas associadas a este cenário, cf. Sec. 5). De acordo com a Eq. (25), o valor do potencial sobre o horizonte de eventos depende do valor de $q Q$ : é zero, se $q Q=0$, e positivo (negativo), se $q Q>0(<0)$.

A posição radial da órbita circular estável mais interna, $r_{\text {ocei }}$, de partículas carregadas, é obtida da solução da equação

$$
\left.\frac{d^{2} V_{q}}{d r^{2}}\right|_{r_{o c e i}}=0
$$




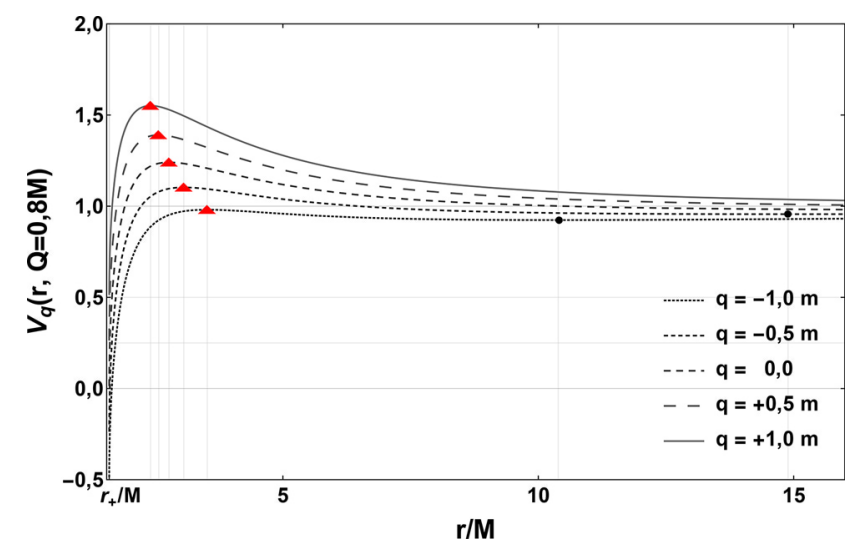

Figura 7: Potencial para partículas carregadas, dado pela Eq. (25), com $l / m=5 M, Q=0,8 M$ e alguns valores de q. Os triângulos vermelhos e os discos pretos são pontos de máximo e mínimo, respectivamente. Os pontos de mínimo, para as cargas $q \geq 0$ listadas na figura, estão localizados em $r>16 M$.

para uma órbita circular genérica, na posição radial $r$, temos

$$
\begin{array}{r}
6 \frac{l^{4} Q^{4}}{m^{4}}-20 M \frac{l^{4} Q^{2}}{m^{4}} r+2 \frac{q Q}{m} r\left(\left(\frac{l^{2}}{m^{2}}+r^{2}\right) f(r) r^{2}\right)^{3 / 2}+ \\
+3 r^{6}\left(\frac{l^{2}}{m^{2}}+Q^{2}+M^{2}\right)-6 M r^{5}\left(3 \frac{l^{2}}{m^{2}}+Q^{2}\right)+ \\
+2 r^{4}\left(\frac{l^{4}}{m^{4}}+7(l / m)^{2} Q^{2}+11 M^{2} \frac{l^{2}}{m^{2}}+Q^{4}\right)+ \\
-6 M r^{3}\left(2 \frac{l^{4}}{m^{4}}+5 \frac{l^{2}}{m^{2}} Q^{2}\right)+ \\
+r^{2}\left(9 \frac{l^{4}}{m^{4}} Q^{2}+15 M^{2} \frac{l^{4}}{m^{4}}+9 \frac{l^{2}}{m^{2}} Q^{4}\right)-2 M r^{7}=0
\end{array}
$$

onde o momento angular $l$, que pode ser $l_{+}$ou $l_{-}$, é dado pela Eq. (21). Se $q=0$ e $|Q|=0(=M)$, obtemos $r_{\text {ocei }}=6 M(=4 M)$, conforme apresentado na Fig. 4 Outros valores para $r_{\text {ocei }}$ estão listados na Tab. 1

Tabela 1: Alguns valores aproximados da posição $r_{o c e i}$, para órbitas de partículas com carga $q$ no entorno de buracos negros com carga $Q$.

\begin{tabular}{lll}
\hline Carga $Q$ & $\begin{array}{l}\text { Carga-teste } q / m \\
(-q / m)\end{array}$ & $\begin{array}{l}\text { Posição } \\
r_{\text {ocei }} / M\end{array}$ \\
\hline \multirow{3}{*}{$Q \rightarrow 0,2 M$} & $0,2(-0,2)$ & $\approx 5,935(\approx 5,941)$ \\
& $0,5(-0,5)$ & $\approx 5,943(\approx 5,946)$ \\
& $1,0(-1,0)$ & $\approx 5,960(\approx 5,960)$ \\
\hline \multirow{3}{*}{$Q \rightarrow 0,8 M$} & $0,2(-0,2)$ & $\approx 4,837(\approx 4,957)$ \\
& $0,5(-0,5)$ & $\approx 4,800(\approx 5,069)$ \\
& $1,0(-1,0)$ & $\approx 5,272(\approx 5,272)$ \\
\hline \multirow{3}{*}{$Q \rightarrow 1,0 M$} & $0,2(-0,2)$ & $\approx 4,832(\approx 4,159)$ \\
& $0,5(-0,5)$ & $\approx 3,558(\approx 4,384)$ \\
& $1,0(-1,0)$ & $\approx 3,000(\approx 4,732)$ \\
\hline
\end{tabular}

\section{Trajetórias de Partículas-Teste}

\subsection{Geodésicas Nulas}

Podemos escolher o parâmetro afim $\lambda$ de forma a escrevermos a Eq. (16), para partículas não-massivas, como

$$
\dot{r}^{2}+\frac{f(r)}{r^{2}}=\frac{1}{b^{2}},
$$

onde

$$
b \equiv l / E
$$

é o parâmetro de impacto da geodésica nula.

A quantidade $b$, definida na Eq. (29), pode ser melhor entendida a partir da seguinte análise da Fig. 8 Em um espaço-tempo plano (e.g. descrito pela Eq. (5) $\mathrm{com} M=$ $|Q|=0$ ), uma partícula massiva livre segue a trajetória retilínea, assinalada como $C^{\prime}$ na Fig. 8 Neste caso, temse $b=b^{\prime}$, sendo $b^{\prime}$ a distância de maior aproximação da partícula em relação a origem $(x=0, y=0)$. O momento angular da partícula, denotado por $\vec{l}$, é 43

$$
\vec{l}=\frac{m}{\sqrt{1-v^{2}}} \vec{r} \times \vec{v}
$$

onde $\vec{v} \equiv d \vec{r} / d t$ é a velocidade da partícula em relação à origem 17 . Podemos extrair da Fig. 8 a velocidade relativa $\vec{v}$ e a posição $\vec{r}$ da partícula em relação a origem, quais sejam

$$
\vec{v}=(v, 0) \quad \vec{r}=(x, b) .
$$

Deste modo, temos que a magnitude do momento angular $l$ é dada por

$$
l=\frac{m}{\sqrt{1-v^{2}}} v b
$$

Pela Eq. (32), pode-se reescrever a quantidade $b \mathrm{em}$ termos do momento angular $l$, da velocidade $v$ e da
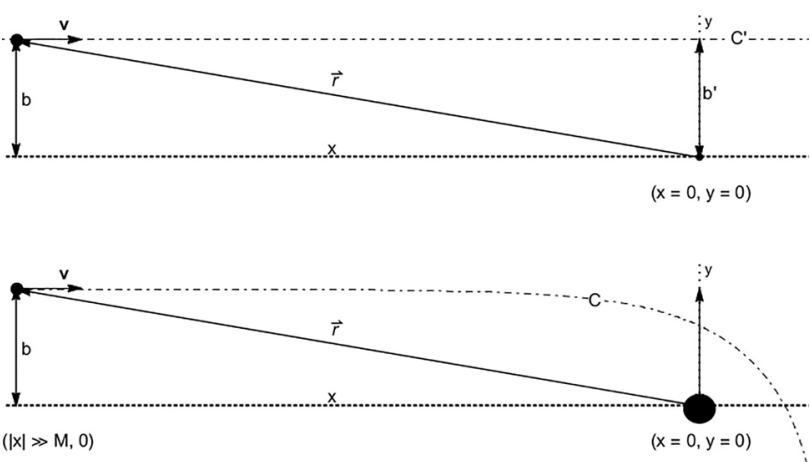

Figura 8: Trajetória $C^{\prime}$ de uma partícula em um espaço-tempo plano (acima), e trajetória $C$ de uma partícula em um espaçotempo curvo (abaixo). O espaço-tempo curvo em análise é caracterizado por um objeto central na origem do sistema de coordenadas. As duas coordenadas espaciais são $x$ (abcissa) e $y$ (ordenada), conforme indicado.

${ }^{17}$ Nota-se que consideramos o observador em repouso no infinito, com tempo próprio $t$, definido no elemento de linha dado pela Eq. [5. 
energia da partícula, $E \equiv m d t / d \tau=m / \sqrt{1-v^{2}}$, qual seja

$$
b=\frac{l}{E v} .
$$

Para o caso de uma partícula não-massiva, cuja trajetória é uma geodésica nula, a velocidade relativa é dada por $v=c=1$, obtendo-se a Eq. 29.

Em espaços-tempos curvos assintoticamente planos (e.g., o espaço-tempo de RN), a Eq. 29 continua sendo válida se a quantidade $b$ for definida no infinito [5]. Neste caso, a partícula segue a trajetória curvilínea assinalada como $C$ na Fig. 8. Deste modo, podemos definir a quantidade $b$, da Eq. 29), como a distância, no infinito, entre a geodésica e o eixo das abcissas do sistema de coordenadas, conforme ilustrado na Fig. 8

Analisando os extremos do potencial efetivo, dado pela Eq. (18) $\operatorname{com} \epsilon=0$, obtemos a posição radial da geodésica circular nula, localizada fora do horizonte de eventos do buraco negro, qual seja

$$
r_{c}=\frac{3}{2} M+\sqrt{\frac{9}{4} M^{2}-\frac{8}{4} Q^{2}} .
$$

Nas situações em que $|Q|=0(=M)$, obtemos $r_{c}=3 M$ $(=2 M)$, como apresentado na Fig. 4

Uma partícula, proveniente do infinito espacial, pode descrever uma geodésica que tende assintoticamente à forma circular em $r_{c}$. Neste caso, o parâmetro de impacto é denominado crítico $\left(b \equiv b_{c}\right)$. Pela Eq. (28), tem-se que

$$
b_{c}=\frac{r_{c}}{\sqrt{f\left(r_{c}\right)}} \text {. }
$$

As geodésicas com parâmetro de impacto menor (maior) que este valor são absorvidas (espalhadas) pelo buraco negro, como ilustrado nas Figs. 9 e 10 [obtidas usando-se a Eq. [28].

Desta maneira, a seção de choque de absorção 18 do buraco negro, no limite geométrico, para um espaço-

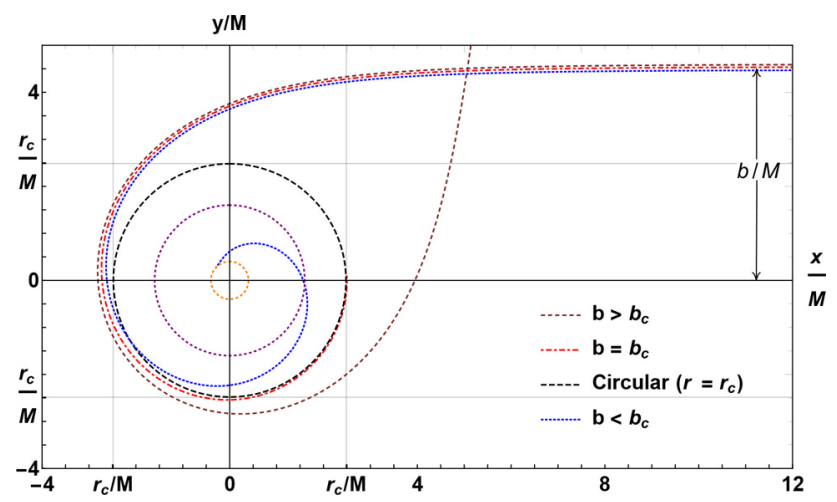

Figura 9: Geodésicas nulas para diferentes valores do parâmetro de impacto. O buraco negro possui carga $|Q|=0,8 M$. Os círculos pontilhadas são os horizontes de eventos, localizado em $r_{+}=1,6 M$, e de Cauchy, localizado em $r_{-}=0,4 M$.

\footnotetext{
18 A seção de choque de absorção é dada pelo fluxo de partículas absorvidas dividido pela densidade de corrente incidente [4].
}

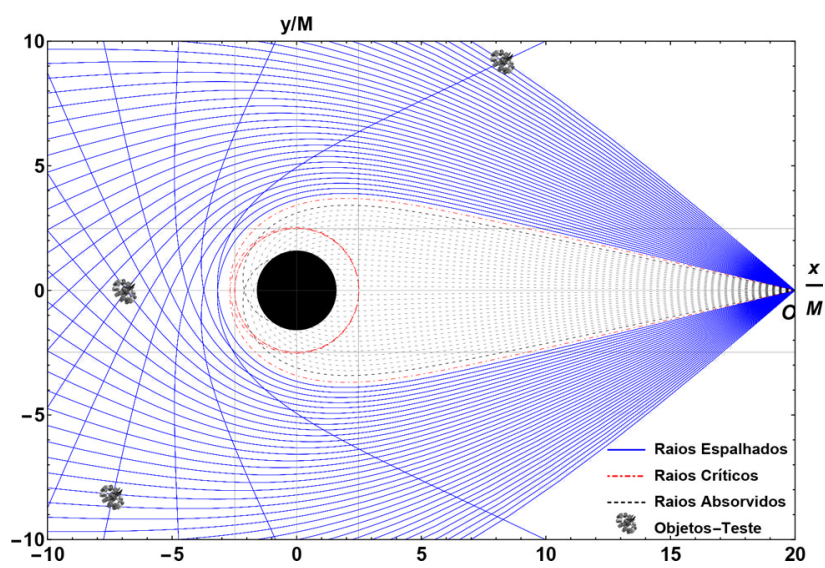

Figura 10: Efeito do encurvamento da trajetória de partículas não-massivas partindo da posição do observador $O$ em várias direções. Estas trajetórias também podem ser entendidas como raios de luz emitidos por objetos-teste no entorno do buraco negro e que chegam ao observador $O$. O disco preto, de raio $r=1,6 M$, denota a região interna ao horizonte de eventos do buraco negro com $|Q|=0,8 M$.

tempo esfericamente simétrico, é dada por 45

$$
\sigma_{a b s} \equiv \pi b_{c}^{2}
$$

Devido ao encurvamento das trajetórias tipo-luz, um observador distante percebe uma imagem de fundo, por detrás do buraco negro, distorcida e/ou multiplicada (efeito por vezes referido como lente gravitacional) ao redor do horizonte de eventos, como pode ser identificado na Fig. 10. Os raios de luz emitidos por objetos-teste, em diferentes regiões do plano da Fig. 10 podem chegar ao observador $O$ por caminhos distintos, podendo contornar o buraco negro. Os objetos-teste sobre o eixo que liga o centro do buraco negro e o observador $O$, devido à simetria esférica, aparecem ao longo de uma circunferência concêntrica ao horizonte de eventos do buraco negro (anel de Einstein).

Ainda devido ao encurvamento da luz pelo campo gravitacional, o tamanho aparente dos objetos, como o Sol, visto por um observador distante dele, é maior que seu tamanho real, pois a luz que emerge de sua superfície tem sua trajetória encurvada pelo campo gravitacional do Sol [35. A carga elétrica de um objeto contribui para diminuir seu tamanho aparente, porque as geodésicas são menos defletidas se o objeto gerador do campo gravitacional possuir carga elétrica.

Na Fig. 11, ilustramos geodésicas nulas provenientes do infinito com parâmetro de impacto fixo, incidindo em buracos negros de RN, com diferentes valores de $|Q|$. Escolhemos $b=3 \sqrt{3} M$, que é o valor crítico do parâmetro de impacto para um buraco negro de Schwarzschild. As geodésicas são menos encurvadas à medida que a carga do buraco negro aumenta de $|Q| / M=0$ (buraco negro de Schwarzschild) até $|Q| / M=1$ (buraco negro extremamente carregado). 


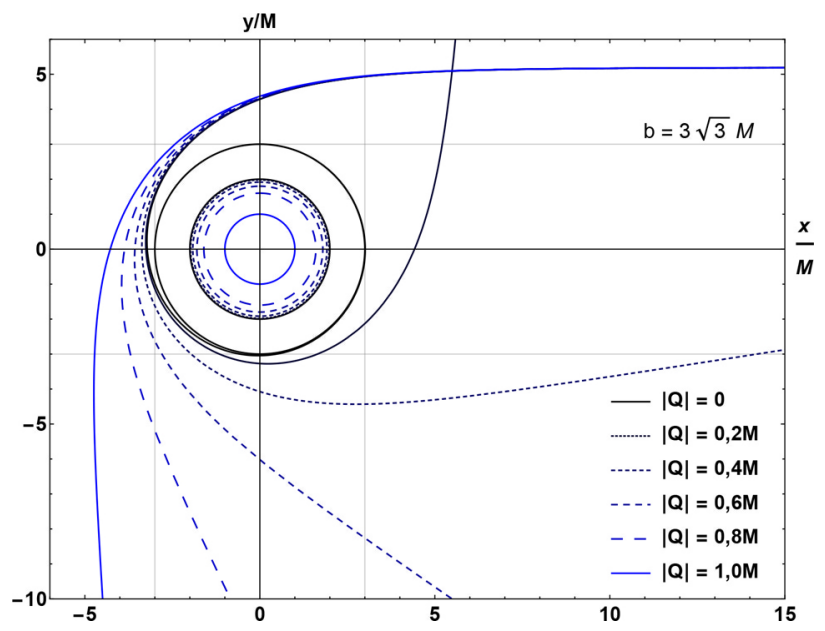

Figura 11: Geodésicas nulas com parâmetro de impacto $b=$ $3 \sqrt{3} M$, no entorno de buracos negros estáticos com carga variando de zero a $M$. Os círculos centrais são os respectivos horizontes de eventos, $r_{+}$, dos buracos negros.

\subsection{Partículas-Teste Massivas Neutras}

As partículas massivas neutras seguem trajetórias geodésicas tipo-tempo. As órbitas dessas partículas são ligadas, quando $E<m$, ou não ligadas, quando $E \geq m$. No último caso, a partícula tem energia suficiente para escapar do campo gravitacional. No caso $E=m$, a partícula apresenta um estado de repouso no infinito. Na Eq. (24), se $E=m$, obtemos a órbita circular ligada mais interna possível, que ocorre em $r_{o c l i}$. A posição $r_{o c l i}$ varia com a carga do buraco negro, como mostrado na Fig. 4.

$\mathrm{Na}$ Fig. 12, ilustramos as trajetórias de primeiro e segundo tipo, as quais se aproximam assintoticamente da órbita circular instável em $r_{o c l i}$. Uma partícula neutra nesta órbita circular instável, ao sofrer uma perturbação radialmente para fora (dentro), passa a seguir uma órbita de primeiro (segundo) tipo, escapando para o infinito (caindo no buraco negro).

\subsection{Partículas-Teste Massivas Carregadas}

Nos cenários de buracos negros carregados, as partículas eletricamente carregadas não seguem trajetórias geodésicas do espaço-tempo de RN, devido à força de Lorentz entre as partículas-teste e o buraco negro. Esta força contribui para o encurvamento das trajetórias. Como consequência, quanto maior for o produto $q Q$, menor é a seção de choque de absorção do buraco negro para essas partículas 46]. Quando $q Q>0$, a força de Lorentz compete com a interação gravitacional, defletindo menos as trajetórias, em comparação às trajetórias de partículas neutras. Por outro lado, se as cargas da partícula $(q)$ e do buraco negro $(Q)$ têm sinais opostos, a seção de choque de absorção para essas partículas se torna maior do que para as partículas neutras, devido à força de Lorentz ser atrativa neste caso [46].
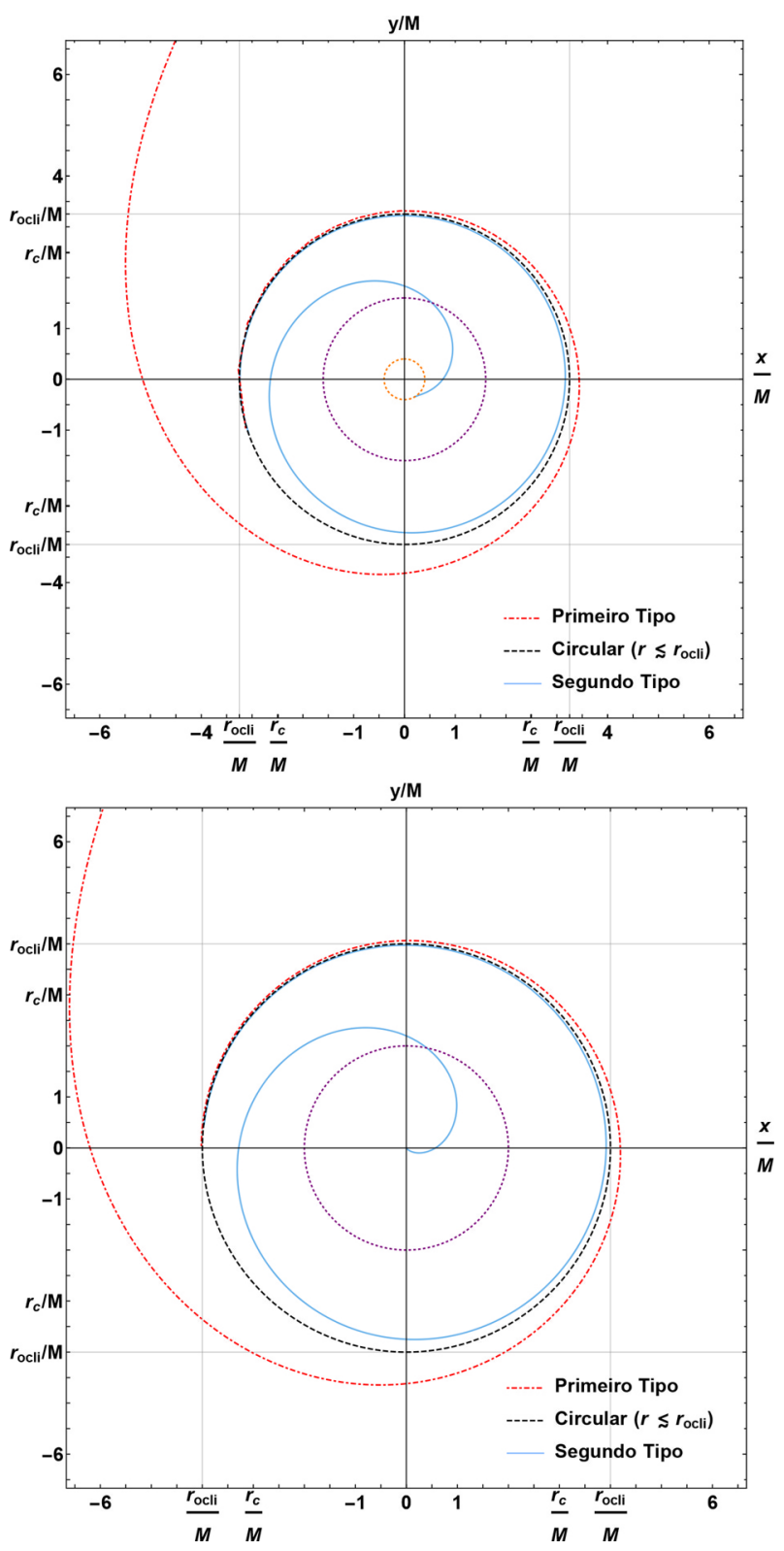

Figura 12: Trajetórias de primeiro e segundo tipo que tendem a órbitas circulares instáveis. Os buracos negros possuem $|Q| / M=$ 0,8 (acima) e $|Q| / M=0$ (abaixo). Os círculos pontilhados representam os horizontes de eventos (externo) e de Cauchy (interno) do buraco negro.

Na Fig. 13, ilustramos trajetórias de partículas carregadas (linhas tracejadas) assim como a trajetória de uma partícula neutra (linha sólida) que tende a uma trajetória circular ao redor do buraco negro positivamente carregado. As partículas com carga de sinal contrário ao da carga do buraco negro são absorvidas pelo buraco negro, enquanto que as partículas com carga de sinal igual ao da carga do buraco negro são espalhadas pelo mesmo. 


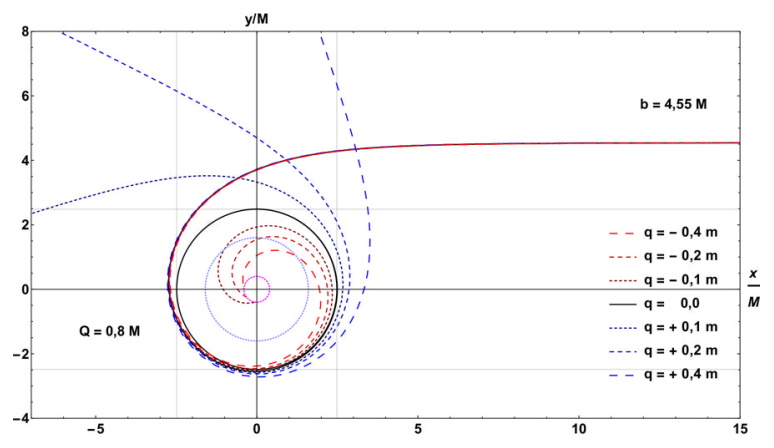

Figura 13: Trajetórias de partículas-teste carregadas com parâmetro de impacto $b=4,55 \mathrm{M}$ e com vários valores de $q$. O buraco negro possui $Q=0,8 M$. Os círculos pontilhados representam os horizontes do buraco negro de RN.

\section{Discussões Finais}

Investigamos o espaço-tempo esfericamente simétrico de um buraco negro estático carregado [buraco negro de Reissner-Nordström (RN)] e descarregado (buraco negro de Schwarzschild). Analisamos as trajetórias de partículas neutras e eletricamente carregadas no entorno destes objetos.

Como consequência das simetrias dos espaços-tempos estudados, as trajetórias de partículas são governadas por uma única equação diferencial para a coordenada radial. Comparamos o potencial efetivo de partículas neutras no contexto da Teoria da Relatividade Geral e da gravitação newtoniana. Analisamos, também, as órbitas circulares e suas condições de estabilidade.

Em comparação a um buraco negro descarregado de mesma massa, o buraco negro carregado deflete menos as geodésicas. Quando aumentamos o parâmetro de carga $|Q|$ do buraco negro, a sua sombra, a seção de choque de absorção para geodésicas nulas e as posições radiais das geodésicas circulares $r_{c}$ (geodésica nula circular), $r_{\text {ocli }} \mathrm{e}$ $r_{o c e i}$ (geodésicas tipo-tempo circulares ligada e estável mais interna, respectivamente) tornam-se menores 19

As partículas eletricamente carregadas não descrevem geodésicas do espaço-tempo de um buraco negro carregado, por interagirem com o campo elétrico do buraco negro via força de Lorentz. Analisamos as órbitas circulares e suas condições de estabilidade também para partículas carregadas. As trajetórias das partículas com carga $q$ de sinal oposto (igual) a do buraco negro, são mais (menos) encurvadas do que as trajetórias das partículas neutras. Isto ocorre devido à ação da força de Lorentz entre a partícula e o buraco negro carregado.

Como concluído na Ref. 46], quanto maior o produto $q Q$, menor é a seção de choque de absorção do buraco negro de RN para partículas carregadas. A absorção de partículas com carga de mesmo sinal que a carga do buraco negro é desfavorecida se $q Q>M m$, uma vez que a repulsão eletrostática será maior que a atração

\footnotetext{
${ }^{19}$ Para uma análise das forças de maré no espaço-tempo de RN, ver Ref. 42.
}

gravitacional a grandes distâncias e a partícula só será absorvida se for projetada contra o buraco negro com certa velocidade inicial mínima. Esta desigualdade é facilmente satisfeita para partículas subatômicas como o elétron ou o pósitron, e o próton ou o antipróton, com o sinal apropriado para $Q$. Isto está associado ao processo de neutralização dos buracos negros carregados 47].

As diferenças entre as geometrias de RN e de Schwarzschild, quando analisadas, ajudam a compreender a influência da carga elétrica na geometria do espaço-tempo. Analisar as trajetórias de partículas em um espaço-tempo associado a um buraco negro de RN, como o que foi feito neste trabalho, nos ajuda a explorar e entender melhor algumas características da Teoria da Relatividade Geral.

\section{AGRADECIMENTOS}

Agradecemos a Hernando Quevedo Cubillos e a Parthasarathi Majumdar, por comentários e sugestões a este trabalho, durante o VI Amazonian Workshop on Black Holes and Analogue Models of Gravity, ocorrido em Belém do Pará. Agradecemos também a Jorge Castiñeiras Rodríguez por discussões estimulantes sobre o tema deste artigo. O presente trabalho foi realizado com apoio da Coordenação de Aperfeiçoamento de Pessoal de Nível Superior - Brasil (CAPES) - Código de Financiamento 001, e pelo Conselho Nacional de Desenvolvimento Científico e Tecnológico (CNPq).

\section{Referências}

[1] H. Reissner, Ann. Phys. 355, 106 (1916).

[2] G. Nordström, Proc. Ac. Amsterdam 20, 1238 (1918).

[3] K. Schwarzschild, Sitzungsber. Preuss. Akad. Wiss. Berlin (Math. Phys.) 10, 189 (1916).

[4] A. Saa, Rev. Bras. Ens. Fis. 38, e4201 (2016).

[5] R.M. Wald, General Relativity (University of Chicago Press, Chicago, 1984).

[6] S.M. Carroll, Spacetime and geometry. An introduction to general relativity (Pearson Education Limited, São Francisco, 2014).

[7] S. Chandrasekhar e J.B. Hartle, Proc. Royal Soc. Lond. 384, 301 (1982).

[8] S. Chandrasekhar, The Mathematical Theory of Black Holes (Oxford University Press, Oxford, 1983).

[9] R.A. Matzner e N. Zamorano, Phys. Rev. D 19, 2821 (1979).

[10] M. Simpson e R. Penrose, Int. J. Theor. Phys. 7, 183 (1973).

[11] R. Narayan, New J. Phys. 7, 199 (2005).

[12] K. Akiyama, A. Alberdi, W. Alef, K. Asada, R. Azulay, A. Baczko, D. Ball, M. Baloković, J. Barrett, D. Bintley et al., Astrophys. J. Lett. 875, L1 (2019).

[13] K. Akiyama, A. Alberdi, W. Alef, K. Asada, R. Azulay, A. Baczko, D. Ball, M. Baloković, J. Barrett, D. Bintley et al., Astrophys. J. Lett. 875, L2 (2019).

[14] K. Akiyama, A. Alberdi, W. Alef, K. Asada, R. Azulay, A. Baczko, D. Ball, M. Baloković, J. Barrett, D. Bintley et al., Astrophys. J. Lett. 875, L3 (2019). 
[15] K. Akiyama, A. Alberdi, W. Alef, K. Asada, R. Azulay, A. Baczko, D. Ball, M. Baloković, J. Barrett, D. Bintley et al., Astrophys. J. Lett. 875, L4 (2019).

[16] K. Akiyama, A. Alberdi, W. Alef, K. Asada, R. Azulay, A. Baczko, D. Ball, M. Baloković, J. Barrett, D. Bintley et al., Astrophys. J. Lett. 875, L5 (2019).

[17] K. Akiyama, A. Alberdi, W. Alef, K. Asada, R. Azulay, A. Baczko, D. Ball, M. Baloković, J. Barrett, D. Bintley et al., Astrophys. J. Lett. 875, L6 (2019).

[18] P.V.P. Cunha e C.A.R. Herdeiro, Gen. Relativ. Gravit. 50, $42(2018)$

[19] A. Einstein, Sitzungsber. Preuss. Akad. Wiss. Berlin (Math. Phys.) 3, 831 (1915).

[20] A. Einstein, Sitzungsber. Preuss. Akad. Wiss. Berlin (Math. Phys.) 3, 844 (1915).

[21] F.W. Dyson, A.S. Eddington e C. Davidson, Phil. Trans. R. Soc. Lond. 220, 291 (1920).

[22] L.C.B. Crispino e D.J. Kennefick, Nat. Phys. 15, 416 (2019).

[23] L.C.B. Crispino e M.C. de Lima, Rev. Bras. Ens. Fis. 38, e4203 (2016).

[24] I.I. Shapiro, Science 157, 806 (1967).

[25] R.V. Pound e G.A. Rebka Jr, Phys. Rev. Lett. 4, 337 (1960).

[26] R. Abuter, A. Amorim, N. Anugu, M. Bauböck, M. Benisty, J. P. Berger, N. Blind, H. Bonnet, W. Brandner, A. Buron et al., Astron. Astrophys. 615, L15 (2018).

[27] D. Pugliese, H. Quevedo e R. Ruffini, Phys. Rev. D 83, 024021 (2011).

[28] E.P.T. Liang, Phys. Rev. D 9, 3257 (1974).

[29] P. Pradhan e P. Majumdar, Phys Lett. A 375, 474 (2011).

[30] D. Pugliese, H. Quevedo e R. Ruffini, Phys. Rev. D 83, 104052 (2011).

[31] D. Pugliese, H. Quevedo e R. Ruffini, Eur. Phys. J. C 77, 206 (2017).

[32] C.W. Misner, K.S. Thorne e J.A. Wheeler, Gravitation (W.H. Freeman and Company, São Francisco, 1973).

[33] R. d'Inverno, Introducing Einstein's Relatvity (Oxford University Press, Oxford, 1999).

[34] L.D. Landau e E.M. Lifshitz, The classical theory of fields (Pergamon Press, Oxford, 1971).

[35] M.P. Hobson, G. Efstathiou e A.N. Lasenby, General Relativity. An Introduction for Physicists (Cambridge University Press, Cambridge, 2006).

[36] J.D. Jackson, Classical Electrodynamics (John Wiley \& Sons, Hoboken, 1998).

[37] R.C. Henry, Astrophys. J. 535, 350 (2000).

[38] R. Ruffini, Phys. Lett. B 41, 334 (1972).

[39] E. Poisson, Living Rev. Relativ. 7, 6 (2004).

[40] K.R. Symon, Mechanics (Addison-Wesley, Massachusetts, 1971).

[41] S.T. Thornton e J.B. Marion, Dinâmica clássica de partículas e sistemas (CENGAGE Learning, São Paulo, 2016).

[42] L.C.B. Crispino, A. Higuchi, L.A. Oliveira e E.S. de Oliveira, Eur. Phys. J. C 76, 168 (2016).

[43] R. Resnick, Introdução à relatividade especial (Editora Polígono, São Paulo, 1971).

[44] H. Friedrich, Scattering Theory (Springer, Berlim, 2013).

[45] L.C.B. Crispino, A. Higuchi e E.S. Oliveira, Phys. Rev. D 80, 104026 (2009).
[46] C.L. Benone e L.C.B. Crispino, Phys. Rev. D 93, 024028 (2016).

[47] G.W. Gibbons, Commun. Math. Phys. 44, 245 (1975). 